\title{
The Gibbard random dictatorship theorem: a generalization and a new proof
}

\author{
Arunava Sen
}

Received: 24 November 2010 / Accepted: 10 January 2011 / Published online: 1 March 2011

(C) The Author(s) 2011. This article is published with open access at SpringerLink.com

\begin{abstract}
This paper proves stronger versions of the Gibbard random dictatorship theorem using induction on the number of voters. It shows that when there are at least three voters, every random social choice function defined on a domain satisfying a Free Triple at the Top property and satisfying a weak form of strategy-proofness called Limited-Comparison Strategy-proofness and Unanimity, is a random dictatorship provided that there are at least three alternatives. The weaker notion of strategy-proofness requires truth-telling to maximize a voter's expected utility only for a limited class of von Neumann-Morgenstern utility representations of the voter's true preference ordering. In the case of two voters, an even weaker condition on the domain and a weaker notion of strategy-proofness are sufficient for the random dictatorship result.
\end{abstract}

Keywords Gibbard's random dictatorship theorem · Free Triple at the Top · Limited comparison strategy-proofness

\section{JEL Classification D71}

\section{Introduction}

Randomization is a natural way to resolve conflicts of interest in group-decision problems. In the standard random voting model, voters report their ordinal preferences

\footnotetext{
Salvador Barberà is one of the pioneers in probabilistic mechanism design theory. He introduced me to the subject when I visited the Economics Department of the Universitat Autònoma de Barcelona in 1987. My own ideas in this area and on the theory of strategy-proofness in general have been strongly shaped by his work and by numerous discussions I have had with him over the years. It is a particular pleasure to be able to contribute to a volume celebrating his 65 th birthday. I would also like to thank Debasis Mishra, Souvik Roy and two anonymous referees of the journal for their comments on the paper.
}

A. Sen $(\bowtie)$

Indian Statistical Institute, New Delhi, India

e-mail: asen@isid.ac.in 
over candidates or alternatives to the mechanism designer who then proposes a lottery over the alternatives. Does this approach significantly expand the class of incentive-compatible outcome functions relative to the deterministic case? In particular, can we escape from the negative conclusions of the Gibbard-Satterthwaite Theorem (Gibbard 1973; Satterthwaite 1975)?

A fundamental issue in the formulation of incentive-compatibility in this model is the way in which voters evaluate lotteries. An obvious approach is to assume that voters are expected utility maximizers. ${ }^{1}$ However, since voters only reveal ordinal preference information, the notion of incentive-compatibility still remains unspecified. For instance, how does a mechanism designer ascertain whether truth-telling maximizes a voter's expected utility if she does not know the voter's von NeumannMorgenstern (vN-M) utility function? In a seminal paper Gibbard (1977), proposed a definition according to which a random social choice function is dominant-strategy incentive-compatible or strategy-proof if telling the truth maximizes a voter's expected utility for every vN-M representation of his ordinal preferences. This is equivalent to requiring the truth-telling lottery to stochastically dominate all lotteries obtained by misrepresentation of preferences. It is easy to verify that the convex combination of strategy-proof random social choice functions is also strategy-proof; the set of such functions can thus be characterized by its extreme points. The paper demonstrates that these extreme points are special random social choice functions called unilaterals and duples. An immediate corollary of this powerful result is that a strategy-proof random social choice function that satisfies the additional property of unanimity must be a random dictatorship. Unanimity is a weak form of efficiency that requires an alternative ranked unanimously best by all voters be chosen with certainty. Thus, the extreme points of the set of unanimous, strategy-proof random social choice functions are in fact, the set of unanimous, strategy-proof deterministic social choice functions. ${ }^{2}$

This paper has a two-fold purpose. The first is to provide a direct proof of the random dictatorship result based on induction on the number of voters. The overall structure of the argument is a generalization of the proof of the Gibbard-Satterthwaite Theorem in Sen (2001). ${ }^{3}$ The second and more important purpose is to generalize the random dictatorship result by showing that it holds under much weaker assumptions than the ones used in Gibbard (1977) and Duggan (1996).

The paper shows that the strategy-proofness requirement can be weakened significantly; in particular, a much weaker notion of stochastic dominance suffices. Consider the case where there are $m$ alternatives $a_{1}, a_{2}, \ldots, a_{m}$ with $m \geq 3$ and suppose that an arbitrary voter has a preference ordering where $a_{j}$ strictly better than $a_{j+1}$, $j=1, \ldots, m-1$. Suppose that truth-telling by this voter leads to the lottery $\lambda$ ( $\lambda_{j}$ is the probability of choosing $a_{j}$ ) when other voters make a particular profile of announcements. Suppose that a misrepresentation yields the lottery $\lambda^{\prime}$. The Gibbard definition of strategy-proofness requires $\sum_{j=1}^{k} \lambda_{j} \geq \sum_{j=1}^{k} \lambda_{j}^{\prime}$ for all $k=1, \ldots, m-1$.

\footnotetext{
1 For a related though different approach, see Barberà (1977).

2 There is a substantial literature that follows the Gibbard approach to defining strategy-proofness in ordinal voting models_-see for instance, Barberà (1979), Ehlers et al. (2002) and Dutta et al. (2002).

3 An alternative proof of the random dictatorship result based on geometric arguments can be found in Duggan (1996).
} 
The paper's definition of strategy-proofness, limited comparison strategy-proofness or LCSP only requires that the inequality hold for $k=1,2,3$. Thus truth-telling and misrepresentation could result in non-comparable lotteries, i.e. there could exist vN-M utility representations of true preferences where a misrepresentation gives higher expected utility than truth-telling. However as long as truth-telling maximizes expected utility for utility representations where the top $k$ alternatives for $k=1,2,3$ get "significantly" higher utility than the other alternatives (for instance, if the top alternatives have utilities arbitrarily close to one while the remaining alternatives have utilities arbitrarily close to zero), the random dictatorship result holds.

The other generalization of the random dictatorship theorem is in the specification of the domain of preferences. The Gibbard result assumes that the domain consists of all antisymmetric orderings of the elements of the set of alternatives. The main result in the paper establishes random dictatorship for an domain of antisymmetric orderings satisfying the Free Triple at the Top or FTT assumption. These are domains where the three top ranked alternatives in an ordering can be chosen arbitrarily. The complete domain clearly satisfies the FTT assumption but an FTT domain can be "much smaller" than the complete domain.

An interesting question which arises is whether the LCSP and FTT conditions can be weakened further. Unfortunately, the paper is unable to provide a complete answer to this question. However, in the special case of two voters, a partial answer can be obtained. Theorem 2 demonstrates that in this case, weaker conditions on strategy-proofness and the domain suffice. The strategy-proofness condition requires the stochastic dominance condition to hold only for the top two alternatives, i.e. for $k=1,2$ instead of $k=1,2,3$ in the requirement of LCSP described earlier. The domain also only requires the two top ranked alternatives to be chosen arbitrarily. It is easy to see that the strategy-proofness condition cannot be weakened further. Suppose that the strategy-proofness condition only required that the probability of a voter's top-ranked alternative not increase by misrepresentation. Consider a random social choice function which places arbitrary probabilities on voters top-ranked alternatives (i.e. the probability distributions can depend on the announced preference profile). It is easy to verify that this function is strategy-proof in the sense described above. Whether strategy-proofness defined for the top two (rather than the top three) alternatives is sufficient for random dictatorship, is an open question.

The question about domains where strategy-proofness (say, in the sense of Gibbard 1977 ) in conjunction with unanimity implies random dictatorship is perhaps more subtle than the one regarding the appropriate definition of strategy-proofness. Aswal et al. (2003) and Chatterji and Sen (2010) have identified sufficient conditions on domains such that all deterministic strategy-proof social choice functions satisfying unanimity, defined over these domains, is dictatorial. A natural conjecture is that such domains are also random dictatorial and in general, that all dictatorial domains are random dictatorial. This appears to be a difficult question and is beyond the scope of this paper.

Both Gibbard's original proof and the proof in Duggan (1996) rely heavily on the complete domain and strategy-proofness assumptions. Their arguments cannot be adapted to prove the results in the paper. The proofs in the paper run along the following lines. First an independent proof for the two voter case is given. Then a "cloning" of voters argument is used to show that an $N,(N \geq 3)$ voter random social 
choice function is an $N-1$ voter random dictatorship involving $N-2$ of the original voters and an artificially created "cloned" voter. Finally, the two-voter result is used to decompose the probability weight assigned to the "cloned" voter.

The paper is organized as follows. The next section describes the model and the random dictatorship theorem while Sect. 3 contains the results of the paper and the proofs. The final section concludes.

\section{Preliminaries and Gibbard's random dictatorship theorem}

Let $I=\{1, \ldots, N\}$ denote a finite set of voters. Let $A$ denote a finite set of alternatives where $|A|=m$. We let $\mathcal{L}(A)$ denote the set of probability distributions or lotteries over the elements of the set $A$.

Each voter $i \in I$ is assumed to have a preference ordering $P_{i}$ over the elements of the set $A$. We assume throughout that $P_{i}$ is a linear ordering, i.e $P_{i}$ is complete, reflexive, transitive and antisymmetric. For any pair of elements $a, b \in A, a P_{i} b$ will signify the following: " $a$ is strictly better than $b$ according to $P_{i}$ ". For any ordering $P_{i}$ and integer $k=1, \ldots, m$, let $r_{k}\left(P_{i}\right) \in A$ denote the $k$ th ranked alternative in $P_{i}$, i.e. $\left|\left\{a \in A: a P_{i} r_{k}\left(P_{i}\right)\right\}\right|=k-1$. A preference profile is a $N$-tuple $P \equiv\left(P_{1}, \ldots, P_{N}\right)$. In other words, a preference profile (or simply, a profile) $P$ is a list of preference orderings, one for each voter. For any profile $P$ and voter $i$, let $P_{-i}$ denote the $N-1$ voter profile $\left(P_{1}, \ldots, P_{i-1}, P_{i+1}, \ldots, P_{N}\right)$. Finally, let $\mathbb{P}$ denote the set of all linear orderings over the elements of the set $A$.

Definition 1 Let $\mathbb{D} \subset \mathbb{P}$. A Random Social Choice Function (RSCF) is a map $\varphi$ : $\mathbb{D}^{N} \rightarrow \mathcal{L}(A)$.

For any profile $P \in \mathbb{D}^{N}, \varphi(P)$ is a probability distribution over $A$. The probability that this distribution assigns to alternative $a \in A$ will be denoted by $\varphi_{a}(P)$. Of course, $\varphi_{a}(P) \geq 0$ and $\sum_{a \in A} \varphi_{a}(P)=1$.

In the standard mechanism design framework, a voter's preference ordering $P_{i}$ is assumed to be private information and must be elicited from the voter. The goal of the theory is to characterize social choice functions that are incentive-compatible, i.e. provide voters appropriate incentives to reveal their private information truthfully. In models where the outcome of voting is probabilistic, there are several notions of incentive-compatibility. We first describe the approach of Gibbard (1977) which is, in fact the standard approach in probabilistic voting theory.

Definition 2 A utility function $u: A \rightarrow \Re$ represents the ordering $P_{i}$ over $A$ if for all $a, b \in A$,

$$
\left[a P_{i} b\right] \Leftrightarrow[u(a)>u(b)]
$$

We let $\mathbb{U}\left(P_{i}\right)$ denote the set of utility functions that represent $P_{i}$. 
Definition 3 A $\operatorname{RSCF} \varphi: \mathbb{D}^{N} \rightarrow \mathcal{L}(A)$ is strategy-proof if, for all $i \in I$, for all $P \in \mathbb{D}^{N}$, for all $\bar{P}_{i} \in \mathbb{D}$ and all $u \in \mathbb{U}\left(P_{i}\right)$, we have

$$
\sum_{a \in A} u(a) \varphi_{a}\left(P_{i}, P_{-i}\right) \geq \sum_{a \in A} u(a) \varphi_{a}\left(\bar{P}_{i}, P_{-i}\right) .
$$

A RSCF is strategy-proof if at every profile no voter can obtain a higher expected utility by deviating from her true preference ordering than she would if she announced her true preference ordering, irrespective of the announcements of the other voters. Here, expected utility is computed with respect an arbitrary utility representation of her true preferences. It is well-known that this is equivalent to requiring that the probability distribution from truth-telling stochastically dominates the probability distribution from misrepresentation in terms of a voter's true preferences. This is stated formally below.

For any $i \in I, P_{i} \in \mathbb{D}$ and $k=1, \ldots, m$, let $B\left(k, P_{i}\right)=\left\{b \in A: b P_{i} r_{k}\left(P_{i}\right)\right\} \cup$ $\left\{r_{k}\left(P_{i}\right)\right\}$, i.e. $B\left(k, P_{i}\right)$ denotes the set of alternatives that are weakly preferred to the $k$ th ranked alternative in $P_{i}$.

Definition $4 \mathrm{~A} \operatorname{RSCF} \varphi: \mathbb{D}^{N} \rightarrow \mathcal{L}(A)$ is strategy-proof if for all $i \in I$, for all $P \in \mathbb{D}^{N}$, for all $\bar{P}_{i} \in \mathbb{D}$ and for all $k=1, \ldots, m-1$, we have

$$
\sum_{b \in B\left(k, P_{i}\right)} \varphi_{b}\left(P_{i}, P_{-i}\right) \geq \sum_{b \in B\left(k, P_{i}\right)} \varphi_{b}\left(\bar{P}_{i}, P_{-i}\right) .
$$

In the proofs of the results in the paper, it will often be convenient to describe situations where a RSCF is manipulable or not strategy-proof. In particular, $\varphi$ is manipulable by voter $i$ at profile $P$ via $\bar{P}_{i}$ if there exists an integer $k \in\{1, \ldots, m-1\}$ such that $\sum_{b \in B\left(k, P_{i}\right)} \varphi_{b}\left(\bar{P}_{i}, P_{-i}\right)>\sum_{b \in B\left(k, P_{i}\right)} \varphi_{b}(P)$.

One of the goals of this paper is to introduce weaker notions of strategy-proofness. For this purpose, it is important to observe that a smaller class of utility representations of $P_{i}$ is sufficient for Definition 3. This restricted class is described below.

Fix $P_{i} \in \mathbb{D}$ and let $k \in\{1, \ldots, m\}$. Let $\mathbb{U}^{k}\left(P_{i}\right)$ denote the set of utility functions $u: A \rightarrow \Re$ defined as follows. Let

$$
u\left(r_{s}\left(P_{i}\right)\right)= \begin{cases}1-(s-1) \delta & \text { if } s \leq k \\ (m-s) \delta & \text { if } s>k\end{cases}
$$

where $0<\delta<\frac{1}{m-2}$. It is easy to verify that all functions in $\mathbb{U}^{k}\left(P_{i}\right)$ represent $P_{i}$ for the specified range of $\delta$. Moreover all utility functions in $\mathbb{U}^{k}\left(P_{i}\right)$ have the property that the utility of the top $k$-alternatives can be chosen to be arbitrarily close to one, while those of the remaining alternatives can be chosen to be arbitrarily close to zero.

Let $\overline{\mathbb{U}}\left(P_{i}\right)=\cup_{k=1}^{m} \mathbb{U}^{k}\left(P_{i}\right)$. Definition 3 can be restated as below.

Definition 5 A $\operatorname{RSCF} \varphi: \mathbb{D}^{N} \rightarrow \mathcal{L}(A)$ is strategy-proof if, for all $i \in I$, for all $P \in \mathbb{D}^{N}$, for all $\bar{P}_{i} \in \mathbb{D}$ and all $u \in \overline{\mathbb{U}}\left(P_{i}\right)$, we have

$$
\sum_{a \in A} u(a) \varphi_{a}\left(P_{i}, P_{-i}\right) \geq \sum_{a \in A} u(a) \varphi_{a}\left(\bar{P}_{i}, P_{-i}\right) .
$$


The weaker notions of strategy-proofness used in the paper can be equivalently formulated by imposing further restrictions on the set $\bar{U}\left(P_{i}\right)$. This will be seen in the next section.

Throughout the paper, attention is restricted to RSCFs satisfying the property of unanimity. This requires that an alternative that is first-ranked by all voters in any profile be selected with probability one in that profile. Unanimity is an innocuous assumption in the context of deterministic social choice functions; every deterministic, strategy-proof social choice function whose range is the set $A$, satisfies unanimity. The counterpart of this claim in the probabilistic model is the following: if, for all alternatives there exists a profile where the alternative is chosen with probability one, then a strategy-proof RSCF satisfies unanimity.

Definition 6 A RSCF $\varphi: \mathbb{D}^{N} \rightarrow \mathcal{L}(A)$ satisfies unanimity if for all $P \in \mathbb{D}^{N}$ and $a \in A$,

$$
\left[a=r_{1}\left(P_{i}\right) \text { for all } i \in I\right] \Rightarrow\left[\varphi_{a}(P)=1\right] \text {. }
$$

A salient class of RSCFs is the class of random dictatorships.

Definition 7 The $\operatorname{RSCF} \varphi: \mathbb{D}^{N} \rightarrow \mathcal{L}(A)$ is a random dictatorship if there exist nonnegative real numbers $\beta_{i}, i \in I$ with $\sum_{i \in I} \beta_{i}=1$ such that for all $P \in \mathbb{D}^{N}$ and $a \in A$,

$$
\varphi_{a}(P)=\sum_{\left\{i: r_{1}\left(P_{i}\right)=a\right\}} \beta_{i}
$$

In a random dictatorship, each voter $i$ gets weight $\beta_{i}$ where the sum of these $\beta_{i}$ 's is one. At any profile, the probability assigned to an alternative $a$ is simply the sum of the weights of the voters whose maximal element is $a$. A random dictatorship is clearly strategy-proof for any domain; by manipulation, a voter can only transfer weight from her most-preferred to a less-preferred alternative. A fundamental result in Gibbard (1977) states that the converse is also true for the complete domain $\mathbb{P}$.

Theorem 1 (Gibbard 1977) Assume $m \geq 3$. A RSCF $\varphi: \mathbb{P}^{N} \rightarrow \mathcal{L}(A)$ is strategyproof and satisfies unanimity if and only if it is a random dictatorship.

Theorem 1 above is an immediate corollary of a more general result proved in Gibbard (1977). In particular, Gibbard's result does not assume unanimity. There are strategy-proof RSCFs that violate unanimity and the general characterization is considerably more difficult. Our goal in this paper is to demonstrate that the random dictatorship result can be obtained with a weaker notion of strategy-proofness and a more restricted domain.

\section{The results}

Weaker notions of strategy-proofness are introduced. They are equivalent to requiring that truth-telling maximize expected utility for more restricted classes of utility representations. 
Definition 8 The $\operatorname{RSCF} \varphi: \mathbb{D}^{N} \rightarrow \mathcal{L}(A)$ is Weak Limited-Comparison Strategyproof (WLCSP) if for all $i \in I$, for all $P \in \mathbb{D}^{N}$, for all $\bar{P}_{i} \in \mathbb{D}$ and $k=1,2$ we have

$$
\sum_{b \in B\left(k, P_{i}\right)} \varphi_{b}\left(P_{i}, P_{-i}\right) \geq \sum_{b \in B\left(k, P_{i}\right)} \varphi_{b}\left(\bar{P}_{i}, P_{-i}\right) .
$$

A slightly stronger version of WLCSP is the following requirement.

Definition 9 The $\operatorname{RSCF} \varphi: \mathbb{D}^{N} \rightarrow \mathcal{L}(A)$ is Limited-Comparison Strategy-proof (LCSP) if for all $i \in I$, for all $P \in \mathbb{D}^{N}$, for all $\bar{P}_{i} \in \mathbb{D}$ and $k=1,2,3$ we have

$$
\sum_{b \in B\left(k, P_{i}\right)} \varphi_{b}\left(P_{i}, P_{-i}\right) \geq \sum_{b \in B\left(k, P_{i}\right)} \varphi_{b}\left(\bar{P}_{i}, P_{-i}\right) .
$$

The WLCSP can be restated in terms of truth-telling maximizing expected utility within a limited class of utility representations of true preferences.

Definition $10 \mathrm{~A} \operatorname{RSCF} \varphi: \mathbb{D}^{N} \rightarrow \mathcal{L}(A)$ is WLCSP if, for all $i \in I$, for all $P \in \mathbb{D}^{N}$, for all $\bar{P}_{i} \in \mathbb{D}$ and all $u \in \mathbb{U}^{1}\left(P_{i}\right) \cup \mathbb{U}^{2}\left(P_{i}\right)$, we have

$$
\sum_{a \in A} u(a) \varphi_{a}\left(P_{i}, P_{-i}\right) \geq \sum_{a \in A} u(a) \varphi_{a}\left(\bar{P}_{i}, P_{-i}\right) .
$$

Similarly,

Definition $11 \mathrm{~A} \operatorname{RSCF} \varphi: \mathbb{D}^{N} \rightarrow \mathcal{L}(A)$ is LCSP if, for all $i \in I$, for all $P \in \mathbb{D}^{N}$, for all $\bar{P}_{i} \in \mathbb{D}$ and all $u \in \mathbb{U}^{1}\left(P_{i}\right) \cup \mathbb{U}^{2}\left(P_{i}\right) \cup \mathbb{U}^{3}\left(P_{i}\right)$, we have

$$
\sum_{a \in A} u(a) \varphi_{a}\left(P_{i}, P_{-i}\right) \geq \sum_{a \in A} u(a) \varphi_{a}\left(\bar{P}_{i}, P_{-i}\right) .
$$

Note that WLSCP is equivalent to strategy-proofness in the case $m=3$ and LCSP is equivalent to strategy-proofness in the cases $m=3$ and $m=4$. However, they are strictly weaker than strategy-proofness for larger $m$. In other words, it is possible to satisfy LCSP and yet find utility representations of a voter's true ordering such that she obtains a higher expected utility by misrepresentation.

The following domain restrictions are considered.

Definition 12 A domain $\mathbb{D}$ is a Free Pair at the Top or (FPT) domain if, for all $a, b \in A$, there exists $P_{i} \in \mathbb{D}$ such that $r_{1}\left(P_{i}\right)=a$ and $r_{2}\left(P_{i}\right)=b$.

Definition 13 A domain $\mathbb{D}$ is a Free Triple at the Top or (FTT) domain if, for all $a, b, c \in A$, there exists $P_{i} \in \mathbb{D}$ such that $r_{1}\left(P_{i}\right)=a, r_{2}\left(P_{i}\right)=b$ and $r_{3}\left(P_{i}\right)=c$.

Note that a FTT domain is an FPT domain and that the complete domain $\mathbb{P}$ is an FTT domain. Of course, FPT and FTT domains with $m(m-1)$ and $m(m-1)(m-2)$ orderings can be constructed which are much smaller than $m$ ! which is the size of the complete domain. These domains are discussed at greater length in Aswal et al. (2003). 
Theorem 2 Assume $m \geq 3$ and let $\mathbb{D}$ be a FPT domain. A RSCF $\varphi: \mathbb{D}^{2} \rightarrow \mathcal{L}(A)$ satisfies unanimity and WLCSP if and only if it is a random dictatorship.

Proof We only prove necessity. Let $I=\{1,2\}$ and let $\mathbb{D}$ be a FPT domain. Assume that $\varphi: \mathbb{D}^{2} \rightarrow \mathcal{L}(A)$ satisfies unanimity and WLCSP.

Lemma 1 Let $P=\left(P_{1}, P_{2}\right)$ be such that $r_{1}\left(P_{1}\right) \neq r_{1}\left(P_{2}\right)$. Then $\left[\varphi_{a}\left(P_{1}, P_{2}\right)>\right.$ $0] \Rightarrow\left[a \in\left\{r_{1}\left(P_{1}\right), r_{1}\left(P_{2}\right)\right\}\right]$.

Proof Suppose noti.e. suppose that there exists $P_{1}, P_{2}$ and $a, b \in A$ such that $r_{1}\left(P_{1}\right)=$ $a \neq b=r_{1}\left(P_{2}\right)$ and $\varphi_{a}\left(P_{1}, P_{2}\right)+\varphi_{b}\left(P_{1}, P_{2}\right)<1$. Let $\alpha=\varphi_{a}\left(P_{1}, P_{2}\right)$ and $\beta=$ $\varphi_{b}\left(P_{1}, P_{2}\right)$. Let $P_{1}^{\prime}=\left(\begin{array}{c}a \\ b \\ \vdots\end{array}\right)$ and $P_{2}^{\prime}=\left(\begin{array}{c}b \\ a \\ \vdots\end{array}\right)$. Then WLCSP implies $\varphi_{a}\left(P_{1}^{\prime}, P_{2}\right)=\alpha$. Also $\varphi_{a}\left(P_{1}^{\prime}, P_{2}\right)+\varphi_{b}\left(P_{1}^{\prime}, P_{2}\right)=1$; otherwise voter 1 will manipulate via $P_{2}$, thereby obtaining probability one on $b$ by unanimity. Hence $\varphi_{b}\left(P_{1}^{\prime}, P_{2}\right)=1-\alpha$. Note that WLCSP also implies $\varphi_{b}\left(P_{1}^{\prime}, P_{2}^{\prime}\right)=\varphi_{b}\left(P_{1}^{\prime}, P_{2}\right)=1-\alpha$ and $\varphi_{a}\left(P_{1}^{\prime}, P_{2}^{\prime}\right)=\alpha$.

By a symmetric argument, $\varphi_{b}\left(P_{1}^{\prime}, P_{2}^{\prime}\right)=\varphi_{b}\left(P_{1}, P_{2}^{\prime}\right)=\beta$ and $\varphi_{a}\left(P_{1}^{\prime}, P_{2}^{\prime}\right)=1-\beta$. Comparing the probabilities on $a$ and $b$ given by $\varphi$ at the profile $\left(P_{1}^{\prime}, P_{2}^{\prime}\right)$, it follows that $\alpha+\beta=1$ contradicting the earlier conclusion.

Lemma 2 Let $P, \bar{P} \in \mathbb{P}^{2}$ be such that $r_{1}\left(P_{1}\right)=a \neq b=r_{1}\left(P_{2}\right)$ and $r_{1}\left(\bar{P}_{1}\right)=c \neq$ $d=r_{1}\left(\bar{P}_{2}\right)$. Then $\left[\varphi_{a}(P)=\varphi_{c}(\bar{P})\right]$ and $\left[\varphi_{b}(P)=\varphi_{d}(\bar{P})\right]$.

Proof Let $P_{1}=\left(\begin{array}{c}a \\ \vdots\end{array}\right)$ and $P_{2}=\left(\begin{array}{c}b \\ \vdots\end{array}\right)$. Let $\hat{P}$ be an arbitrary profile where $r_{1}\left(\hat{P}_{1}\right)=$ $a$ and $r_{1}\left(\hat{P}_{2}\right)=b$. Then WLCSP implies that $\varphi_{a}\left(\hat{P}_{1}, P_{2}\right)=\varphi_{a}\left(P_{1}, P_{2}\right)$. Lemma 1 implies $\varphi_{b}\left(\hat{P}_{1}, P_{2}\right)=\varphi_{b}\left(P_{1}, P_{2}\right)$. Now changing voter 2 's ordering from $P_{2}$ to $\hat{P}_{2}$ and applying the same arguments, it follows that $\varphi_{a}\left(\hat{P}_{1}, \hat{P}_{2}\right)=\varphi_{a}\left(P_{1}, P_{2}\right)$ and $\varphi_{b}\left(\hat{P}_{1}, \hat{P}_{2}\right)=\varphi_{b}\left(P_{1}, P_{2}\right)$.

Assume that $c \neq b$. The argument in the previous paragraph implies that it can be assumed without loss of generality that $c$ is the second ranked outcome at $P_{1}$ (if $a$ and $c$ are distinct), i.e. it can be assumed that $P_{1}=\left(\begin{array}{c}a \\ c \\ \vdots\end{array}\right)$. Let $\bar{P}_{1}=\left(\begin{array}{c}c \\ a \\ \vdots\end{array}\right)$. Then WLCSP implies $\varphi_{a}\left(\bar{P}_{1}, P_{2}\right)+\varphi_{c}\left(\bar{P}_{1}, P_{2}\right)=\varphi_{a}\left(P_{1}, P_{2}\right)+\varphi_{c}\left(P_{1}, P_{2}\right)$. By Lemma $1, \varphi_{c}\left(P_{1}, P_{2}\right)=\varphi_{a}\left(\bar{P}_{1}, P_{2}\right)=0$. Hence $\varphi_{a}\left(P_{1}, P_{2}\right)=\varphi_{c}\left(\bar{P}_{1}, P_{2}\right)$ while $\varphi_{b}\left(P_{1}, P_{2}\right)=\varphi_{b}\left(\bar{P}_{1}, P_{2}\right)$. Assume $b \neq d$. Switching voter 2 's preferences from $P_{2}$ to $\bar{P}_{2}$ and applying the same argument as above, it follows that $\varphi_{c}\left(\bar{P}_{1}, P_{2}\right)=\varphi_{c}\left(\bar{P}_{1}, \bar{P}_{2}\right)$ while $\varphi_{b}\left(\bar{P}_{1}, P_{2}\right)=\varphi_{d}\left(\bar{P}_{1}, \bar{P}_{2}\right)$.

The arguments above can deal with all cases except the case where $c=b$ and $d=a$. Since $m \geq 3$, there exists $x \in A$ distinct from $a$ and $b$. Let $\tilde{P}_{1}$ be such that $r_{1}\left(\tilde{P}_{1}\right)=x$. From earlier arguments $\varphi_{a}\left(P_{1}, P_{2}\right)=\varphi_{x}\left(\tilde{P}_{1}, \bar{P}_{2}\right)$ and $\varphi_{b}\left(P_{1}, P_{2}\right)=\varphi_{a}\left(\tilde{P}_{1}, \bar{P}_{2}\right)$. 
Applying these arguments again, it can be inferred that $\varphi_{x}\left(\tilde{P}_{1}, \bar{P}_{2}\right)=\varphi_{b}\left(\bar{P}_{1}, \bar{P}_{2}\right)$ and $\varphi_{a}\left(\tilde{P}_{1}, \bar{P}_{2}\right)=\varphi_{a}\left(\bar{P}_{1}, \bar{P}_{2}\right)$ establishing the Lemma.

Lemmas 1 and 2 above establish that $\varphi$ is a random dictatorship. Observe that the existence of the preference orderings that have been used are guaranteed by the assumption that $\mathbb{D}$ is an FPT domain.

The next result extends Theorem 2 albeit under stronger assumptions.

Theorem 3 Assume $m \geq 3, N \geq 3$ and let $\mathbb{D}$ be a FTT domain. A RSCF $\varphi: \mathbb{D}^{N} \rightarrow$ $\mathcal{L}(A)$ satisfies unanimity and LCSP if and only if it is a random dictatorship.

Proof Once again only necessity is proved. Let $N \geq 3$ be an integer. Let $\varphi: \mathbb{D}^{N} \rightarrow$ $\mathcal{L}(A)$ satisfy unanimity and LCSP. It will be shown that $\varphi$ is a random dictatorship.

We prove the result by induction. Assume that for all integers $K<N$, the following statement is true:

Induction Hypothesis (IH): Assume $m \geq 3$ and let $\mathbb{D}$ be an FTT domain. If $\varphi: \mathbb{D}^{K} \rightarrow$ $\mathcal{L}(A)$ satisfies unanimity and LCSP, then it is a random dictatorship.

Let $\hat{I}=\{\hat{1}, 3, \ldots, N\}$ be a set of voters where $3, \ldots, N \in I$. Define a RSCF $g: \mathbb{D}^{N-1} \rightarrow \mathcal{L}(A)$ for the set of voters $\hat{I}$ as follows: For all $P_{\hat{1}}, P_{3}, \ldots, P_{N} \in \mathbb{D}^{N-1}$,

$$
g\left(P_{\hat{1}}, P_{3}, \ldots, P_{N}\right)=\varphi\left(P_{1}, P_{1}, P_{3}, \ldots, P_{N}\right)
$$

Voter $\hat{1}$ in the RSCF $g$ is obtained by "cloning" voters 1 and 2 in $I$. Thus if voters 1 and 2 in $I$ have a common ordering $P_{1}$, then voter $\hat{1}$ in $\hat{I}$ has ordering $P_{\hat{1}}$.

Lemma 3 The RSCF $g$ is a random dictatorship.

Proof It is trivial to verify that $g$ satisfies unanimity. We will show that $g$ satisfies LCSP so that the result follows by an application of IH. Let $\left(P_{1}, P_{3}, \ldots, P_{N}\right) \in \mathbb{D}^{N-1}$ and let $\bar{P}_{1} \in \mathbb{D}$. Let $k \in\{1,2,3\}$. We have

$$
\begin{aligned}
\sum_{b \in B\left(k, P_{i}\right)} g_{b}\left(P_{\hat{1}}, P_{3}, \ldots, P_{N}\right) & =\sum_{b \in B\left(k, P_{i}\right)} \varphi_{b}\left(P_{1}, P_{1}, P_{3}, \ldots, P_{N}\right) \\
& \geq \sum_{b \in B\left(k, P_{i}\right)} \varphi_{b}\left(\bar{P}_{1}, P_{1}, P_{3}, \ldots, P_{N}\right) \\
& \geq \sum_{b \in B\left(k, P_{i}\right)} \varphi_{b}\left(\bar{P}_{1}, \bar{P}_{1}, P_{3}, \ldots, P_{N}\right) \\
& =\sum_{b \in B\left(k, P_{i}\right)} g_{b}\left(\bar{P}_{\hat{1}}, P_{3}, \ldots, P_{N}\right)
\end{aligned}
$$

If inequality 2 does not hold, voter 1 with ordering $P_{1}$ manipulates $\varphi$ at $\left(P_{1}, P_{1}, \ldots, P_{N}\right)$ via $\bar{P}_{1}$. If inequality 3 does not hold, voter 2 with ordering $P_{1}$ manipulates $\varphi$ at $\left(\bar{P}_{1}, P_{1}, \ldots, P_{N}\right)$ via $\bar{P}_{1}$. Therefore $g$ satisfies LCSP.

Let $\beta, \beta_{3}, \ldots, \beta_{N}$ be the weights associated with the random dictatorship $g$; i.e. $\beta_{i}$, is the weight associated with voter $i, i=3, \ldots, N$ and $\beta$ is the weight associated with voter $\hat{1}$. 
Lemma 4 Let $P \in \mathbb{D}^{N}$ be a an arbitrary profile. Let $a=r_{1}\left(P_{1}\right)$ and $b=r_{1}\left(P_{2}\right)$ and let $\beta^{x} \equiv \sum_{\left\{i \in\{3, \ldots, N\}: r_{1}\left(P_{i}\right)=x\right\}} \beta_{i}$ for all $x \in A$. Then

(i) $\varphi_{a}(P)=\beta+\beta^{a}$ if $a=b$.

(ii) $\varphi_{a}(P)+\varphi_{b}(P)=\beta+\beta^{a}+\beta^{b}$ if $a \neq b$.

(iii) $\varphi_{c}(P)=\beta^{c}$ for all $c \neq a, b$.

Proof We first show $(i)$ and $($ iii $)$ in the case where $a=b$, i.e. $r_{1}\left(P_{1}\right)=r_{1}\left(P_{2}\right)=a$.

We have,

$$
\begin{aligned}
\varphi_{a}\left(P_{1}, P_{2}, P_{3}, \ldots, P_{N}\right) & =\varphi_{a}\left(P_{1}, P_{1}, P_{3}, \ldots, P_{N}\right) \\
& =g_{a}\left(P_{\hat{1}}, P_{3}, \ldots, P_{N}\right) \\
& =\beta+\beta^{a} .
\end{aligned}
$$

Equation 4 follows from the assumption that $\varphi$ is LCSP and the fact that $r_{1}\left(P_{1}\right)=$ $r_{1}\left(P_{2}\right)=a$. This establishes $(i)$.

Now suppose $r_{1}\left(P_{1}\right)=r_{1}\left(P_{2}\right)=a$ but there exists $c \neq a$ such that $\varphi_{c}(P) \neq \beta^{c}$. Suppose $\varphi_{x}(P)<\beta^{x}$ for all $x \neq a$. From part $(i)$ above, it is known that $\varphi_{a}(P)=$ $\beta+\beta^{a}$. Also $\beta+\beta^{a}+\sum_{x \neq a} \beta^{x}=1$ since $g$ is a random dictatorship. Therefore $1=\sum_{y \in A} \varphi_{y}(P)<\beta+\beta^{a}+\sum_{x \neq a} \beta^{x}=1$ which is a contradiction. Hence there exists $c \neq a$ such that $\varphi_{c}(P)>\beta^{c}$.

Let $\bar{P}_{1}=\left(\begin{array}{c}a \\ c \\ \vdots\end{array}\right)$. We know from part $(i)$ above that $\varphi_{a}\left(\bar{P}_{1}, P_{2}, P_{3}, \ldots, P_{N}\right)=$ $\varphi_{a}(P)=\beta+\beta^{a}$. Since $\varphi$ satisfies LCSP, it must be true that $\varphi_{c}\left(\bar{P}_{1}, P_{2}, P_{3}, \ldots, P_{N}\right)>$ $\beta^{c}$. Hence

$$
\begin{aligned}
& \varphi_{a}\left(\bar{P}_{1}, P_{2}, P_{3}, \ldots, P_{N}\right)+\varphi_{c}\left(\bar{P}_{1}, P_{2}, P_{3}, \ldots, P_{N}\right)>\beta+\beta^{a}+\beta^{c} \\
& \quad=g_{a}\left(\bar{P}_{\hat{1}}, P_{3}, \ldots, P_{N}\right)+g_{c}\left(\bar{P}_{\hat{1}}, P_{3}, \ldots, P_{N}\right) \\
& \quad=\varphi_{a}\left(\bar{P}_{1}, \bar{P}_{1}, P_{3}, \ldots, P_{N}\right)+\varphi_{c}\left(\bar{P}_{1}, \bar{P}_{1}, P_{3}, \ldots, P_{N}\right)
\end{aligned}
$$

Therefore voter 2 manipulates $\varphi$ at $\left(\bar{P}_{1}, \bar{P}_{1}, P_{3}, \ldots, P_{N}\right)$ via $P_{2}$. This completes the proof of part (iii) in the case where $a=b$.

Suppose now that $a$ and $b$ are distinct. Let $\bar{P}_{1}=\left(\begin{array}{c}a \\ b \\ \vdots\end{array}\right)$ and $\bar{P}_{2}=\left(\begin{array}{c}b \\ a \\ \vdots\end{array}\right)$ In view of what has been proved earlier, it can be deduced that $\varphi_{a}\left(P_{1}, \bar{P}_{1}, P_{3}, \ldots, P_{N}\right)+$ $\varphi_{b}\left(P_{1}, \bar{P}_{1}, P_{3}, \ldots, P_{N}\right)=\varphi_{a}\left(\bar{P}_{2}, P_{2}, P_{3}, \ldots, P_{N}\right)+\varphi_{b}\left(\bar{P}_{2}, P_{2}, P_{3}, \ldots, P_{N}\right)=$ $\beta+\beta^{a}+\beta^{b}$. Suppose that $\varphi_{a}\left(P_{1}, P_{2}, P_{3}, \ldots, P_{N}\right)+\varphi_{b}\left(P_{1}, P_{2}, P_{3}, \ldots, P_{N}\right) \neq$ $\beta+\beta^{a}+\beta^{b}$. Since $\varphi$ satisfies LCSP, we have $\varphi_{b}\left(P_{1}, \bar{P}_{2}, P_{3}, \ldots, P_{N}\right)=$ $\varphi_{b}\left(P_{1}, P_{2}, P_{3}, \ldots, P_{N}\right)$ and $\varphi_{a}\left(P_{1}, \bar{P}_{2}, P_{3}, \ldots, P_{N}\right)+\varphi_{b}\left(P_{1}, \bar{P}_{2}, P_{3}, \ldots, P_{N}\right)=$ $\varphi_{a}\left(P_{1}, \bar{P}_{1}, P_{3}, \ldots, P_{N}\right)+\varphi_{b}\left(P_{1}, \bar{P}_{1}, P_{3}, \ldots, P_{N}\right)=\beta+\beta^{a}+\beta^{b}$. By a similar argument, $\varphi_{a}\left(\bar{P}_{1}, \bar{P}_{2}, P_{3}, \ldots, P_{N}\right)=\varphi_{a}\left(P_{1}, \bar{P}_{2}, P_{3}, \ldots, P_{N}\right)$ and $\varphi_{a}\left(\bar{P}_{1}, \bar{P}_{2}, P_{3}, \ldots, P_{N}\right)$ $+\varphi_{b}\left(\bar{P}_{1}, \bar{P}_{2}, P_{3}, \ldots, P_{N}\right)=\varphi_{a}\left(\bar{P}_{2}, \bar{P}_{2}, P_{3}, \ldots, P_{N}\right)+\varphi_{b}\left(\bar{P}_{2}, \bar{P}_{2}, P_{3}, \ldots, P_{N}\right)=$ 
$\beta+\beta^{a}+\beta^{b}$. Combining these equalities, it follows that $\varphi_{b}\left(\bar{P}_{1}, \bar{P}_{2}, P_{3}, \ldots, P_{N}\right)=$ $\varphi_{b}(P)$ and $\varphi_{a}\left(\bar{P}_{1}, \bar{P}_{2}, P_{3}, \ldots, P_{N}\right)=\beta+\beta^{a}+\beta^{b}-\varphi_{b}(P)$.

Now first changing voter 1 's ordering from $P_{1}$ to $\bar{P}_{1}$ in the profile $P$ and then changing voter 2 's ordering from $P_{2}$ to $\bar{P}_{2}$ in the profile $\left(\bar{P}_{1}, P_{2}, P_{3}, \ldots, P_{N}\right)$ and using counterparts of earlier arguments, it can be concluded that $\varphi_{a}\left(\bar{P}_{1}, \bar{P}_{2}, P_{3}, \ldots, P_{N}\right)=$ $\varphi_{a}(P)$ and $\varphi_{b}\left(\bar{P}_{1}, \bar{P}_{2}, P_{3}, \ldots, P_{N}\right)=\beta+\beta^{a}+\beta^{b}-\varphi_{a}(P)$. Comparing the probability of $\varphi_{b}\left(\bar{P}_{1}, \bar{P}_{2}, P_{3}, \ldots, P_{N}\right)$ in the two calculations, it follows that $\varphi_{a}(P)+\varphi_{b}(P)=$ $\beta+\beta^{a}+\beta^{b}$. This is a contradiction to the hypothesis that $\varphi_{a}(P)+\varphi_{b}(P) \neq \beta+\beta^{a}+\beta^{b}$.

Finally it is shown that part (iii) holds in the case where $a \neq b$. Suppose this is false. Using part ( $i i)$ above and the earlier argument, an alternative $c \neq a, b$ can be found such that $\varphi_{c}(P)>\beta^{c}$. Since $\mathbb{D}$ is an FTT domain, there exists $\hat{P}_{1}$ and $\hat{P}_{2}$ such that $\hat{P}_{1}=\left(\begin{array}{c}a \\ c \\ b \\ \vdots\end{array}\right)$ and $\hat{P}_{2}=\left(\begin{array}{c}b \\ c \\ a \\ \vdots\end{array}\right)$. Applying LCSP, it can be inferred that $\varphi_{a}\left(\hat{P}_{1}, P_{2}, P_{3}, \ldots, P_{N}\right)=\varphi_{a}(P)$ and $\varphi_{c}\left(\hat{P}_{1}, P_{2}, P_{3}, \ldots, P_{N}\right) \geq \varphi_{c}(P)$. Similarly, $\varphi_{b}\left(\hat{P}_{1}, P_{2}, P_{3}, \ldots, P_{N}\right)=\varphi_{b}\left(\hat{P}_{1}, \hat{P}_{2}, P_{3}, \ldots, P_{N}\right)$ and $\varphi_{c}\left(\hat{P}_{1}, \hat{P}_{2}, P_{3}, \ldots, P_{N}\right) \geq$ $\varphi_{c}\left(\hat{P}_{1}, P_{2}, \ldots, P_{N}\right)$. Using part $(\mathrm{ii})$ above, it follows that $\varphi_{a}\left(\hat{P}_{1}, \hat{P}_{2}, P_{3}, \ldots, P_{N}\right)+$ $\varphi_{b}\left(\hat{P}_{1}, \hat{P}_{2}, P_{3}, \ldots, P_{N}\right)=\beta+\beta^{a}+\beta^{b}$ and $\varphi_{c}\left(\hat{P}_{1}, \hat{P}_{2}, P_{3}, \ldots, P_{N}\right)>\beta^{c}$. Hence $\sum_{\{x=a, b, c\}} \varphi_{x}\left(\hat{P}_{1}, \hat{P}_{2}, P_{3}, \ldots, P_{N}\right)>\beta+\beta^{a}+\beta^{b}+\beta^{c}$.

Let $\tilde{P}_{1}=\left(\begin{array}{c}c \\ a \\ b \\ \vdots\end{array}\right)$ and $\tilde{P}_{2}=\left(\begin{array}{c}c \\ b \\ a \\ \vdots\end{array}\right)$. From earlier arguments, it follows that $\varphi_{x}\left(\tilde{P}_{1}, \tilde{P}_{2}, P_{3}, \ldots, P_{N}\right)=\beta^{x}$ if $x \neq c$ and $\beta+\beta^{c}$ if $x=c$. From LCSP,

$$
\begin{aligned}
\beta+\beta^{a}+\beta^{b}+\beta^{c} & =\sum_{x=a, b, c} \varphi_{x}\left(\tilde{P}_{1}, \tilde{P}_{2}, P_{3}, \ldots, P_{N}\right) \\
& =\sum_{x=a, b, c} \varphi_{x}\left(\hat{P}_{1}, \tilde{P}_{2}, P_{3}, \ldots, P_{N}\right) \\
& =\sum_{x=a, b, c} \varphi_{x}\left(\hat{P}_{1}, \hat{P}_{2}, P_{3}, \ldots, P_{N}\right)
\end{aligned}
$$

However Eq. 5 contradicts the earlier conclusion that $\sum_{x=a, b, c} \varphi_{x}\left(\hat{P}_{1}, \hat{P}_{2}, P_{3}\right.$, $\left.\ldots, P_{N}\right)>\beta+\beta^{a}+\beta^{b}+\beta^{c}$.

The proof is now completed by considering two mutually exhaustive cases.

Case I: $\beta>0$.

Fix $P_{3}, \ldots, P_{N} \in \mathbb{D}$ and define the function $h: \mathbb{D}^{2} \rightarrow \Re^{m}$ below: for all $P_{1}, P_{2} \in$ $\mathbb{D}$ and $a \in A$,

$$
h_{a}\left(P_{1}, P_{2}\right)=\frac{1}{\beta}\left[\varphi_{a}\left(P_{1}, P_{2}, P_{3}, \ldots, P_{N}\right)-\beta^{a}\right]
$$


Lemma 5 The function $h$ is a RSCF and satisfies unanimity and LCSP.

Proof Pick an arbitrary pair of orderings $P_{1}, P_{2} \in \mathbb{D}$. Let $a \in A$. If $r_{1}\left(P_{1}\right)=$ $r_{1}\left(P_{2}\right)=a$, then $\varphi_{a}\left(P_{1}, P_{2}, P_{3}, \ldots, P_{N}\right)=\beta+\beta^{a}$ according to Lemma 4 part $(i)$. Hence $h_{a}\left(P_{1}, P_{2}\right)=1$. Suppose $a=r_{1}\left(P_{1}\right) \neq b=r_{1}\left(P_{2}\right)$. From Lemma 4 part $($ iii $), \varphi_{a}\left(P_{2}, P_{2}, P_{3}, \ldots, P_{N}\right)=\beta^{a}$. It follows from LCSP that $\varphi_{a}\left(P_{1}, P_{2}, \ldots, P_{N}\right) \geq \beta^{a}$. Hence $h_{a}\left(P_{1}, P_{2}\right) \geq 0$. Similarly, $h_{b}\left(P_{1}, P_{2}\right) \geq 0$. If $a \notin\left\{r_{1}\left(P_{1}\right) \cup r_{1}\left(P_{2}\right)\right\}$, then $\varphi_{a}\left(P_{1}, P_{2}, P_{3}, \ldots, P_{N}\right)=\beta^{a}$. Hence $h_{a}\left(P_{1}, P_{2}\right)=0$, i.e $h_{a}\left(P_{1}, P_{2}\right) \geq 0$ for all $a \in A$. Note also that $\sum_{a \in A} \varphi_{a}\left(P_{1}, P_{2}, P_{3}, \ldots, P_{N}\right)=$ $\beta+\sum_{a \in A} \beta^{a}=1$, so that $\sum_{a \in A} h_{a}\left(P_{1}, P_{2}\right)=1$. We have thus shown that $h$ is a RSCF.

It has already been shown that if $r_{1}\left(P_{1}\right)=r_{1}\left(P_{2}\right)=a$, then $h_{a}\left(P_{1}, P_{2}\right)=1$. Therefore $h$ satisfies unanimity.

Let $k \in\{1,2,3\}$ and let $P_{1}, P_{2}, \bar{P}_{1} \in \mathbb{D}$. Then,

$$
\begin{aligned}
\sum_{b \in B\left(k, P_{i}\right)} h_{b}\left(P_{1}, P_{2}\right) & =\frac{1}{\beta}\left[\sum_{b \in B\left(k, P_{i}\right)}\left(\varphi_{b}\left(P_{1}, P_{2}, P_{3}, \ldots, P_{N}\right)-\beta^{b}\right)\right] \\
& \geq \frac{1}{\beta}\left[\sum_{b \in B\left(k, P_{i}\right)}\left(\varphi_{b}\left(\bar{P}_{1}, P_{2}, P_{3}, \ldots, P_{N}\right)-\beta^{b}\right)\right] \\
& =\sum_{b \in B\left(k, P_{i}\right)} h_{b}\left(\bar{P}_{1}, P_{2}\right)
\end{aligned}
$$

where Inequality 6 holds because $\varphi$ is LCSP. Therefore $h$ is LCSP.

Since $\mathbb{D}$ is a FPT domain and $h$ satisfies WLCSP, it follows from Theorem 2 that $h$ is a random dictatorship. Assume that the weights associated with $g$ are $\gamma_{1}$ and $\gamma_{2}$ for voters 1 and 2 respectively. It follows from the definition of $h$ that for all $P_{1}, P_{2} \in \mathbb{D}$ and $a \in A$,

$$
\varphi_{a}\left(P_{1}, P_{2}, P_{3}, \ldots, P_{N}\right)=\sum_{\left\{i \in\{1,2\}: r_{1}\left(P_{i}\right)=a\right\}} \beta \gamma_{i}+\sum_{i \in\{3, \ldots, N\}} \beta_{i}
$$

Therefore $\varphi$ is a random dictatorship with weights $\beta \gamma_{1}, \beta \gamma_{2}, \beta_{3}, \ldots, \beta_{N}$ if the weights for the random dictatorship $h$ do not depend on the initial choice of the profile $\left(P_{3}, \ldots, P_{N}\right)$ for voters $3, \ldots, N$. We shall show that this is indeed the case. In order to show this, it will suffice to show that the weights $\left(\gamma_{1}, \gamma_{2}\right)$ do not change as voter 3 switches unilaterally from $P_{3}$ to an arbitrary $\bar{P}_{3}$ in $\left(P_{3}, \ldots, P_{N}\right)$.

Suppose the weights in $h$ for the profile $\left(\bar{P}_{3}, \ldots, P_{N}\right)$ are $\gamma_{1}^{\prime}, \gamma_{2}^{\prime}$ and $\gamma_{1}^{\prime}>\gamma_{1}$. Let $a=r_{1}\left(P_{3}\right)$ and $b=r_{1}\left(\bar{P}_{3}\right)$. There are two cases to consider. Suppose first that $a=b$. Now pick $P_{1}, P_{2} \in \mathbb{D}$ such that $a=r_{1}\left(P_{1}\right)$ and $c=r_{1}\left(P_{2}\right)$ where $c \neq a$. Then $\varphi_{a}\left(P_{1}, P_{2}, P_{3}, \ldots, P_{N}\right)=\beta \gamma_{1}+\beta^{a}$ while $\varphi_{a}\left(P_{1}, P_{2}, \bar{P}_{3}, \ldots, P_{N}\right)=\beta \gamma_{1}^{\prime}+\beta^{a}$. Clearly voter 3 manipulates at $\left(P_{1}, P_{2}, P_{3}, \ldots, P_{N}\right)$ via $\bar{P}_{3}$.

Suppose $a \neq b$. In view of the properties of $\varphi$ that have already been established, it can be assumed without loss of generality that $b=r_{2}\left(P_{3}\right)$. Let $\bar{\beta}^{a}=$ 
$\sum_{\left\{i \in\{4, \ldots, N\}: r_{1}\left(P_{i}\right)=a\right\}} \beta_{i}$ and let $\bar{\beta}^{b}=\sum_{\left\{i \in\{4, \ldots, N\}: r_{1}\left(P_{i}\right)=b\right\}} \beta_{i}$. Pick $P_{1}, P_{2} \in \mathbb{D}$ such that $a=r_{1}\left(P_{1}\right)$ and $c=r_{1}\left(P_{2}\right)$ where $c \neq a, b$. Then $\sum_{\{x=a, b\}} \varphi_{x}\left(P_{1}, P_{2}, P_{3}, \ldots, P_{N}\right)$ $=\beta \gamma_{1}+\beta_{3}+\bar{\beta}^{a}+\bar{\beta}^{b}$ while $\sum_{\{x=a, b\}} \varphi_{x}\left(P_{1}, P_{2}, \bar{P}_{3}, \ldots, P_{N}\right)=\beta \gamma_{1}^{\prime}+\beta_{3}+\bar{\beta}^{a}+\bar{\beta}^{b}$. Once again voter 3 manipulates at $\left(P_{1}, P_{2}, P_{3}, \ldots, P_{N}\right)$ via $\bar{P}_{3}$.

This completes the proof of random dictatorship in Case I.

Case II: $\beta=0$.

Let $P_{1}, P_{2} \in \mathbb{D}$. Applying Lemma 4 , it follows that $\varphi_{a}\left(P_{1}, P_{2}, P_{3}, \ldots, P_{N}\right)=\beta^{a}$ for all $a \in A$. But this implies that $\varphi$ is a random dictatorship with weights $\beta_{1}=\beta_{2}=0$ and $\beta_{i}, i=3, \ldots, N$. This concludes the proof.

It can be observed that virtually all arguments in the proof of Theorem 3 require only the FPT and WLCSP assumptions. The only place where the full force of FTT and LCSP is used is in one case of part (iii) of Lemma 4. An open question is whether Theorem 3 can be proved using the weaker assumptions.

\section{Conclusion}

This paper provides a new approach to the proof of the Gibbard random dictatorship theorem. This allows a stronger version of the result to be proved.

Open Access This article is distributed under the terms of the Creative Commons Attribution License which permits any use, distribution and reproduction in any medium, provided the original author(s) and source are credited.

\section{References}

Aswal N, Chatterji S, Sen A (2003) Dictatorial domains. Econ Theory 22:45-62

Barberà S (1977) The Manipulation of social choice functions that do not leave "Too Much" to chance. Econometrica 45:1573-1588

Barberà S (1979) Majority and positional voting in a probabilistic framework. Rev Econ Stud 46:379-389 Chatterji S, Sen A (2010) Tops-only domains. Econ Theory (forthcoming)

Duggan J (1996) A geometric proof of Gibbard's random dictatorship theorem. Econ. Theory 7:365-369

Dutta B, Peters H, Sen A (2002) Strategy-proof probabilistic mechanisms in economies with pure public goods. J Econ Theory 106:392-416

Ehlers L, Peters H, Storcken T (2002) Strategy-proof probabilistic decision schemes for one-dimensional single-peaked preferences. J Econ Theory 105:408-434

Gibbard A (1973) The manipulation of voting schemes: a general result. Econometrica 41:587-601

Gibbard A (1977) Manipulation of voting schemes that mix voting with chance. Econometrica 45:665-681

Satterthwaite M (1975) Strategy-proofness and arrow's conditions: existence and correspondence theorems for voting procedures and social welfare functions. J Econ Theory 10:187-217

Sen A (2001) Another direct proof of the Gibbard-Satterthwaite theorem. Economics Letters 70:381-385 